Cahiers de recherches médiévales

Figures mythiques médiévales auX $\mathrm{XIX}^{\mathrm{e}}$ et $\mathrm{XX}$ siècles

\title{
Enfances arthuriennes dans la littérature pour enfants
}

Cécile Boulaire

\section{(2) OpenEdition}

1 Journals

Édition électronique

URL : https://journals.openedition.org/crm/1753

DOI : 10.4000/crm. 1753

ISSN : 1955-2424

Éditeur

Honoré Champion

Édition imprimée

Date de publication : 15 décembre 2004

Pagination : 83-94

ISSN : 1272-9752

\section{Référence électronique}

Cécile Boulaire, «Enfances arthuriennes dans la littérature pour enfants », Cahiers de recherches

médiévales [En ligne], 11 | 2004, mis en ligne le 10 octobre 2007, consulté le 15 décembre 2022. URL

http://journals.openedition.org/crm/1753 ; DOl : https://doi.org/10.4000/crm.1753

Ce document a été généré automatiquement le 15 décembre 2022.

Tous droits réservés 


\title{
Enfances arthuriennes dans la littérature pour enfants
}

\author{
Cécile Boulaire
}

En y réfléchissant, je me suis également dit que - même quand ils le mériteraient les enfants ne deviennent presque jamais aussi célèbres que les grandes personnes. Les livres, les rues, les cratères de la lune, portent rarement ou jamais des noms d'enfants. On parle peu d'eux dans les journaux. À de rares exceptions près, les vies d'enfants sont des vies privées et demeurent inconnues. Comme si l'enfance était seulement un lieu d'attente de la vraie vie. Comme si la vérité d'une personne se révélait seulement lorsqu'elle atteint l'âge adulte. Ce n'est évidemment pas le cas. Il $\mathrm{y}$ a des personnes enfants qui mènent des vies mémorables, des vies captivantes, aventureuses ou étranges. Il y a même des personnes dont la vie d'adulte est bien moins intéressante que ne l'a été leur enfance. ${ }^{1}$

1 Parmi les figures puissantes que le Moyen Âge a léguées à notre époque, les héros arthuriens sont les plus présentes à l'esprit des enfants, loin devant les Charlemagne, Roland ou Saint-Louis qui composaient il y a encore quelques décennies à la fois le Panthéon scolaire et le décor des images offertes aux écoliers méritants. Les réformes des contenus d'enseignements ont progressivement relégué ces grands personnages historiques pour faire advenir l'humble paysan, le serf opprimé - figures historiques elles aussi, mais innombrables et dès lors anonymes. Le Moyen Âge n'a pas pour autant déserté l'imaginaire enfantin, qui ne se nourrit pas, loin s'en faut, à la seule source scolaire. Et les films, séries télévisées, dessins animés, comme les jeux et les jouets, et surtout les romans pour la jeunesse, ont continué à parler aux enfants d'une époque souvent mythique à leurs yeux, et qu'ils perçoivent comme une entité close, à la fois révolue et préservée dans un état immuable, au point que parfois les romans d'heroic fantasy lui supposent une existence parallèle à notre modernité.

2 Alors que la littérature pour enfants au XIX ${ }^{\mathrm{e}}$ siècle isolait encore, dans ce Moyen Âge de chevaliers et de conquêtes, les héros historiques qui avaient "fait » la France, à partir $\mathrm{du}$ début $\mathrm{du} \mathrm{XX}^{\mathrm{e}}$ siècle (et avec beaucoup de retard sur les pays anglo-saxons) on commence à évoquer, à l'intention des petits Français, les aventures empreintes de merveilleux d'Arthur et de ses chevaliers, de Merlin l'enchanteur et de Lancelot. Mais la greffe semble longue à prendre sur un territoire malgré tout profondément marqué par 
le rationalisme d'une part, le moralisme d'autre part. On aimait l'inventivité de Jules Verne, parce qu'elle se parait des atours de la science - plonger dans le merveilleux arthurien, n'était-ce pas soudain revenir à cette littérature invraisemblable dont on s'était tant méfié, allant jusqu'à mettre en garde contre les périls que faisaient courir aux plus jeunes esprits les fariboles véhiculées par les contes de fées? Il faut donc attendre longuement pour que les premières traductions et adaptations de textes arthuriens, importées d'Angleterre, donnent naissance en France à de nouvelles adaptations pour enfants de cette "matière de Bretagne" dont notre patrimoine littéraire est pourtant si riche. Et, même après la Seconde Guerre mondiale, ces récits arthuriens désormais si présents à l'esprit des enfants (notamment grâce à la popularité de grands films de cinéma, comme Les Chevaliers de la table Ronde de Richard Thorpe par exemple) sont pour une grande part des traductions de romans nés en Angleterre ou aux États-Unis. Quelques-uns de ces textes à thématique arthurienne ont retenu mon attention : ceux dans lesquels est évoquée l'enfance des personnages dont la tradition a célébré les exploits en tant qu'adultes. J'en retiendrai cinq ici : The Sword in the stone de Terence Handbury White, publié en 1938, traduit pour la première fois en France en 1965 ; L'Enfance de Merlin, de Claude Sylvain, paru en 1947 ; The Winter Prince, d'Elisabeth E. Wein, paru en 1993, et traduit en 1995 sous le titre Le Bâtard du roi Arthur; Arthur, high king of Britain, de Michael Morpurgo, publié en 1994 et traduit dès 1995 en français ; et enfin, de manière marginale, Le Chien du roi Arthur, d'Odile Weulersse, publié en 1994.

Ces récits pour enfants me semblent, par-delà leurs profondes différences de forme, de ton, de résonance idéologique comme de projet narratif, se situer tous au point de convergence de plusieurs traditions littéraires, voire de plusieurs topoï. Tout d'abord, la tradition médiévale de l'«enfance", qui consiste à reprendre un personnage héroïque existant déjà dans la littérature romanesque et à inventer une nouvelle matière à récit en amont de ses exploits d'adulte héroïque ${ }^{2}$. Ensuite la tradition, héritée du XIX ${ }^{e}$ siècle, des "enfances de grands hommes ", livres destinés aux enfants euxmêmes, et faisant remonter la stature héroïque des grandes figures de l'histoire à une prédestination, à un caractère d'exception qui serait lisible dès l'enfance. Le plus connu est peut-être La Jeunesse des hommes célèbres d'Eugène Müller publié dans la Bibliothèque d'éducation et de récréation de l'éditeur Jules Hetzel, mais on pourrait aussi évoquer par exemple les Enfances célèbres de Louise Colet parues chez Hachette en 1854. Le troisième faisceau convergent concerne la tendance, lisible depuis les années 80 , et largement inspirée par la vogue de l'heroic fantasy, à revisiter parfois jusqu'à l'épuisement la tradition arthurienne et à explorer littérairement les moindres recoins laissés éventuellement vierges par la littérature médiévale. Enfin ces quelques récits adhèrent au topos propre au livre d'enfant, qui veut qu'à de jeunes lecteurs on propose essentiellement des jeunes personnages, non pas dans une optique rétrospective, comme l'est le «récit d'enfance $»^{3}$, mais au contraire dans une vision prospective, qui feint parfois d'ignorer que l'«avenir» des protagonistes essentiels a déjà été abondamment fixé par l'écrit. Comment inventer, lorsqu'on situe sa création au carrefour d'influences si prégnantes? Ces quelques œuvres m'ont pourtant semblé proposer à leurs jeunes lecteurs, par-delà les allégeances aux diverses traditions assumées ou inconscientes, une aventure littéraire à part entière. Enfances de Merlin, d'Arthur ou même du bâtard ordinairement honni Mordred, ces récits au genre quadruplement contraint posent justement la question de la liberté au cœur de la 
contrainte, à travers l'évocation de personnages prédestinés, voués sans échappatoire à un destin héroïque.

4 Le Roi Arthur de Michaël Morpurgo met en scène un enfant contemporain, jamais nommé, qui se perd dans les brumes au cours d'une partie de pêche et, sur le point de se noyer, est recueilli sous les eaux par un vieil homme disert qui se révélera être Arthur. En attendant que les habits du garçon soient secs, Arthur lui fait le récit de sa propre histoire, en commençant par son enfance. Point de liberté ici - du moins pas celle des héros canoniques : Arthur est voué à devenir ce que les récits médiévaux ont fait de lui, et toutes ses tentatives pour fuir cette destinée sont vouées à l'échec. Enfant, lorsqu'il apprend qu'il n'est pas le fils de l'homme qui l'a élevé avec tendresse, il s'échappe, persuadé que la mort mettra fin à son désespoir. Il ne meurt évidemment pas, et un vieux mendiant, rencontré en forêt, lui parle du tapis de jacinthes sauvages sur lequel ils sont assis :

Ne pensez plus à la mort, jeune homme. Ouvrez les yeux et admirez ce qu'une fleur peut faire. Toute la Bretagne pourrait devenir aussi belle que ce bois. Vous pouvez être la première jacinthe, celle par qui tout commence. (Ses yeux noirs me sourirent gentiment, et il ébouriffa le cou de son chien.) Il me suffit de regarder les yeux d'un homme pour voir son âme. Je vois dans la vôtre la graine de la grandeur. Laissez-la germer. (p. 24)

5 La métaphore botanique ne laisse aucune place à l'initiative personnelle : Arthur n'est que le terreau sur lequel, à son corps défendant peut-être, se développera le royaume de Bretagne. Guidé par ce qu'il prend pour du hasard, Arthur, alors qu'il est pressé par son demi-frère de lui fournir une épée, se saisit de celle qu'un rouge-gorge semble lui désigner, une épée brillante plantée dans un bloc de granit. Ce geste, fortuit à ses yeux, le désigne comme roi et sauveur, et dès lors il ne s'appartient plus. L'« enfance » est ici récit d'une cécité; le tragique de cette aventure enfantine réside justement dans cette soumission de l'individu à un itinéraire dont il ne maîtrise ni les détours, ni le sens. Arthur apparaîtra d'ailleurs pareillement victime, dans l'âge adulte, de son désir incestueux pour sa demi-sœur Morgawse ( Je ne pouvais pas m'en douter, mais cette nuit-là, à Caerleon, je semai la graine de ma propre destruction », p. 51) comme de la ruine de son mariage, par la faute de l'amour qui naît entre la reine et Lancelot. Pressentiment et prédiction parcourent le récit, qu'il s'agisse de l'inquiétude de Guenièvre au seuil du mariage comme de la menace que semble incarner le nourrisson porté par Morgawse lors des fêtes nuptiales. Guenièvre, image même de l'individu épris de liberté, redoute les contraintes que font peser sur les êtres institutions, devoirs et raison d'État :

Je désire être votre femme, Arthur, dit-elle, mais je n'ai pas envie de jouer le rôle de reine. Je sais que c'est mon devoir, comme vous devez jouer votre rôle, mais je souhaiterais qu'il en soit autrement. (p. 66)

Arthur, qui est conscient que son devoir est de se plier, non simplement aux obligations du protocole royal, mais à un destin déjà écrit, insiste auprès de Merlin pour connaître l'identité de l'enfant mystérieux :

Vous m'avez promis un jour de ne jamais me prédire l'avenir sans que je l'aie sollicité [...]. Si cet enfant doit jouer un rôle dans mon avenir, alors dites-le moi. (p. 69)

7 À quoi Merlin répond que l'enfant est Mordred, son propre fils, conçu avec sa demisœur. Pour Mordred, pas plus de liberté que pour Arthur : «Il vous trahira. C'est dans ce but qu'il a été conçu, et c'est à cela qu'il consacrera sa vie » (p. 70). À ce tragique des 
destins individuels pris dans les rets d'un implacable destin s'ajoute une construction narrative qui multiplie les figures du double et de la répétition, au point de nier l'historicité et de faire de l'aventure humaine un infini recommencement. En effet, le vieil homme qui recueille le petit pêcheur mouillé au début du roman ressemble trait pour trait, jusqu'au chien qui l'accompagne, au vieux mendiant qu'Arthur enfant avait rencontré en forêt et n'était autre que Merlin, comme si Arthur vieillissant prenait le relais de Merlin - de la même façon que le petit garçon devrait prendre celui d'Arthur. Le vieil homme lui dit en effet: "Quand je te regarde, il me semble revoir l'enfant que j'ai été, rêveur, aventureux" (p.18). Accompagnant Arthur adolescent vers ses responsabilités royales, Merlin poursuit la métaphore botanique :

Aucun arbre, aussi beau soit-il, ne peut être éternel. Un jour, votre arbre se desséchera aussi et mourra. Mais il en restera un gland, un unique gland endormi dans le sol jusqu'au jour où il sera prêt à pousser à nouveau. (p. 38)

8 C'est sans doute ce gland qu'Arthur devenu vieillard offre au jeune garçon, et que celuici emporte chez lui comme preuve matérielle que son aventure sous-marine n'as pas été un rêve. Et l'enfant plante le gland dans son jardin, manière de dire que si le destin étouffe ceux qui doivent s'y soumettre, l'aventure arthurienne est une si puissante incarnation des rêves de grandeur des enfants que ceux-ci rêvent de s'y projeter. C'est aussi le propos tenu dans Le Chien du roi Arthur, où un petit paysan, Oscar, se repaît des récits d'exploits arthuriens que lui raconte le forgeron Cadoc, lui qui a forgé des épées pour les plus grands, Gauvain même. Oscar n'aspire évidemment qu'à devenir chevalier ; or l'occasion s'en présente lorsqu'il est témoin du rapt de Kavall, le chien du roi Arthur-Oscar parviendra évidemment à le libérer et à accéder à la cour de Camelot. Certes, le destin brise les individus, et le roman de Morpurgo, à travers la sensibilité qu'il attribue aux personnages, traduit superbement le tragique de leur situation. Mais dans le même temps l'aventure arthurienne, surtout lorsqu'elle prend la forme d'une « enfance » romanesque, semble être un irrésistible appel à la répétition fantasmatique, par le miracle de la fiction générant rêve et désir : rêve d'héroïsme, désir de chevalerie.

Pourtant la réécriture arthurienne sous la forme d'«enfance " n'est pas vouée à célébrer le fatalisme, fût-il teinté d'héroïsme. D'autres récits bien au contraire prennent prétexte de ces inventions dans le «blanc» du cycle arthurien pour insuffler vie et fantaisie aux cœurs de destinées verrouillées par une tradition romanesque séculaire. Claude Sylvain, pseudonyme de Jeannine Harmand, publie en 1947 le petit album qui s'intitule Contes et légendes du roi Artus. L'enfance de Merlin', peut-être prévu à l'origine pour être le premier d'une série. Il n'est en effet jamais question d'Arthur dans ce court volume, exclusivement consacré à l'enfance miraculeuse de Merlin. Cinq chapitres, rappelant les mirabilia de l'hagiographie médiévale, évoquent cinq épisodes où Merlin, bébé puis petit garçon, fait la preuve de ses dons surnaturels autant que de sa sagesse et de sa malice proverbiales. Le premier chapitre, dans lequel, âgé de quelques heures, il prend la parole pour innocenter sa mère accusée par les juges, évoque effectivement bien des pages de la Légende dorée. Mais le ton ici est débarrassé de tout didactisme : ce Merlin-ci n'est pas un saint, et s'il use de ses dons, c'est par tendresse pour sa mère, harcelée par des voisins jaloux :

- Ne pleurez pas Maman! Ce ne sont que des bêtises! Je vous ferai tant d'honneur un jour que chacun regrettera les mauvaises paroles qu'il vous a dites aujourd'hui. (p. 5) 
- Taisez-vous bavards ! Laissez ma mère en paix, et occupez-vous de ce qui vous regarde ! (p. 7)

- En voilà assez à la longue. Ces mégères m'échauffent les oreilles. Elles osent se moquer de ma mère ! Je vais les changer en pies borgnes. (p. 14)

L'enfant connaît les secrets et les travers de chacun, et c'est avec beaucoup d'astuce qu'il révèle publiquement les petites bassesses d'un juge ou d'un aubergiste, qu'il démasque les usurpateurs dans l'entourage de l'empereur, qu'il parvient à convertir à la prodigalité un gros bourgeois connu pour son avarice. Mais il n'est jamais ennuyeux ou moralisateur, il s'amuse :

Mais celui-ci ne leur répondit pas. Chevauchant une libellule il était allé porter ailleurs sa gaieté et ses conseils. (p. 28)

Merlin n'eut pas plutôt vu cette cour remplie de gens tristes qu'il se mit à rire.

(p. 30)

Toutes les fois qu'il le fallait, Merlin savait punir en se divertissant. (p. 37)

11 C'est donc une "enfance Merlin " légère et facétieuse qui est proposée ici au jeune lecteur, et le plus étrange, c'est qu'elle ne soit rattachée en rien au monde arthurien tel que nous le présentent les versions les plus courantes: l'album s'achève en fait sur "une des plus belles conversions de Merlin», et abandonne le personnage alors qu'il est encore adolescent. En outre, l'illustration elle-même contribue à couper l'album de la vulgate arthurienne, puisque le contexte iconographique est exclusivement romain et antique, quand bien même le texte précise que l'action se situe dans une "bonne ville de Bretagne». Cette apparente contradiction entre le texte et l'image ne manque pas d'intriguer, car plusieurs détails du récit au contraire semblent évoquer un univers moyenâgeux, proche même de celui que mettent en scène les fabliaux : le personnage de l'honnête laboureur; celui du bourgeois avare Gonnebert; les femmes de l'aubergiste, du charron et du menuisier transformées en pies jacassantes. Ce hiatus entre texte et image contribue à plonger le récit dans un hors-temps tout à fait propre au conte. L'auteur ne cherche pas à rattacher les miracles de Merlin à une quelconque transcendance; elle ne cherche pas davantage à faire pressentir au lecteur le futur destin « arthurien » de son jeune personnage. Il semble au contraire qu'elle ait plaisir à s'attarder sur sa jeunesse - une phrase, révélant la bonté de Merlin, donne le sens de cet attachement à l'enfance :

Dès qu'il voulut il sut marcher et grimper. Il bondissait et sautait si légèrement qu'on aurait facilement pu dire qu'il volait ; mais il ne se pressa pas trop de grandir pour laisser à sa mère la joie de profiter de toute son enfance. (p. 21)

Cet album apporte à la somme arthurienne une fraîcheur, une malice inhabituelles, et propose un regard neuf sur un Merlin juvénile qu'on a coutume d'imaginer plutôt en vieux mage barbu. L'« enfance » est ici une manière de libérer personnage et récit, par la fantaisie, d'une tradition parfois écrasante. Un autre auteur, et à une échelle bien supérieure, va insuffler liberté et fantaisie au cœur d'un destin que des siècles de littérature héroïque ont figé : il s'agit de Terence Handbury White et de son Sword in the stone, premier volume, sorti en 1938, d'une réécriture de l'œuvre de Malory qui paraîtra dans son intégralité en 1958 sous le titre The once and future king'. Ce cycle arthurien n'est pas spécifiquement destiné à l'enfance, mais l'œuvre de White comprend au moins un texte pour les enfants, et The Sword in the Stone devient rapidement un classique de l'enfance, au point que Walt Disney s'en empare en 1963 pour en faire un Merlin l'enchanteur qui popularisera les personnages de White au-delà des frontières du monde anglophone. C'est d'ailleurs à la suite du succès du dessin animé que paraitt la première 
traduction française du texte de White, dans une édition expressément destinée aux enfants, puisqu'il s'agit de la Bibliothèque verte 6 . L'action de ce premier volume explore une nouvelle enfance, celle d'Arthur lui-même. Sir Hector, chevalier très british et un peu décati, est soucieux de l'éducation des deux jeunes gens dont il a la charge, son propre fils, Kay, et son pupille, le jeune Art - diminutif du véritable prénom de l'enfant, qu'on ne mentionne qu'à la dernière ligne, comme si le prénom d'Arthur contenait en lui-même la dimension royale et épique d'un personnage que son diminutif, d'ailleurs remplacé le plus souvent par un sobriquet', mettait provisoirement à l'abri du destin... Tandis que Sir Hector et son voisin Sir Grummore Grummursum, autour d'un verre de Porto, discutent des mérites respectifs de l'éducation à l'ancienne, des collèges et des précepteurs, Art, égaré en forêt au cours d'un jeu de chasse, va rencontrer celui qui sera son initiateur, le fantasque Merlin. Après un déjeuner fabuleux, auquel participe Archimède, un hibou qui parle, et tandis que pots et assiettes vont se ranger d'euxmêmes sur les étagères, Merlin annonce à Moustique son intention de le suivre au château pour faire son éducation. Tout le reste du roman est donc consacré aux années d'initiation du jeune Moustique, livré à l'imprévisible Merlin qui met ses dons de magie au service d'une pédagogie inhabituelle. Le vieillard en effet prétend donner à l'enfant la connaissance. Et pour ce faire, nul besoin de livres: Merlin se contente de lui attribuer diverses apparences animales, pour qu'il expérimente des situations variées et ait la connaissance intime du monde qui l'entoure. C'est ainsi que Moustique est successivement transformé en poisson, puis en faucon, en serpent, en oiseau, en blaireau. Mais cette intimité avec la nature n'est qu'un des aspects de son éducation. Merlin souhaite aussi initier Moustique à l'univers fabuleux des créatures livresques, qui ont ici autant de réalité que Sir Hector lui-même. Moustique, rendu invisible par magie, se rend donc dans l'antre du géant Galapas; il rencontre la sorcière Mim; il accompagne Robin Hood et ses compagnons dans une attaque contre les Anthropophages, tous droit sortis d'un bestiaire médiéval ; il bavarde d'égal à égal avec Athéna. Mais Moustique est aussi très imprégné de culture littéraire: il connaît à merveille la littérature courtoise, et rêve de devenir chevalier errant ; il écoute, lors des veillées, le récit des Gesta Romanorum; il entend de la bouche même d'un blaireau le récit (un peu remanié...) de la Création. Au terme des vingt-quatre chapitres, Moustique a donc reçu une éducation très complète. C'est alors que resurgit un fil qui a parcouru discrètement la trame narrative du récit: le roi Uter meurt, et le récit de White est rattrapé par la temporalité inéluctable du texte arthurien canonique : le «blanc» de l'enfance est écoulé, et il est temps pour l'auteur de remettre son héros sur les rails du récit fixé par la tradition. À la suite d'un concours de circonstances, c'est Moustique qui parvient à extraire l'épée de l'enclume. White expédie la cérémonie du couronnement en un très bref chapitre plein de malice, énumérant les cadeaux saugrenus offerts par tous les personnages qui ont traversé le récit. À la dernière page réapparaît Merlin, qui avait disparu depuis l'annonce de la mort du roi. C'est le vieil original qui a le dernier mot, lorsqu'il promet à Moustique de le seconder dans ses responsabilités - et White clôt ainsi son récit sur les mots qui au contraire ouvrent d'autres versions :

«Resterez-vous longtemps près de moi ? demanda-t-il.

- Oui, Moustique ", répondit Merlin.

Il se reprit aussitôt :

« Ou plutôt, je devrais dire... j'aurais dû dire depuis longtemps sans doute : “Oui, roi

Arthur" ». (explicit) 
13 White réalise donc ici un défi : inventer près de 300 pages de récit dont l'amplitude temporelle se déploie dans un des minces interstices vierges laissés par la tradition médiévale. Il dessine un Arthur étrange, juvénile, candide, joyeux, qui jure parfois avec la figure un peu hiératique de certaines versions. Alors qu'on aurait pu imaginer une enfance marquée par les faits d'armes, au contraire la fantaisie et la malice, l'ouverture d'esprit, la curiosité et l'absurde dessinent ici, comme dans le bref album de Claude Sylvain, une manière d'être libre - non de se dérober à son destin, mais d'exister pleinement avant même que de devoir s'y plier.

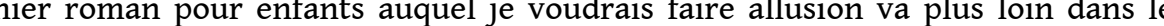
questionnement romanesque sur la liberté. De manière très exceptionnelle, il y est question de Mordred, l'enfant qu'Arthur a conçu d'une union incestueuse avec sa demisœur Morgause. Double liberté que celle qui est offerte au héros ordinairement détesté de l'aventure arthurienne : d'une part parce que l'auteur elle-même s'affranchit de la vulgate arthurienne, inventant partiellement une descendance légitime au couple Arthur-Guenièvre ; d'autre part parce que Mordred va conquérir chèrement, et c'est le sujet du roman, son droit à l'existence, alors même que tout, depuis le tabou de sa filiation jusqu'aux sentiments ambivalents qu'il prête à sa fratrie, le destine à incarner inlassablement le félon. Le récit arthurien, étroitement resserré, est ici l'occasion d'une réflexion romanesque poignante sur le sens de la filiation, sur la recherche d'identité au cœur de la fratrie, sur la jalousie et l'amour.

s'ouvre par un prologue où un narrateur extradiégétique présente, en une courte scène déjà bouleversante, les principaux (et presque uniques) protagonistes du récit: Arthur, dont l'épouse Guenièvre vient de mettre au monde deux jumeaux maladifs, Lohot et Gouvaine ; et Mordred, son premier-né illégitime, déjà taraudé de jalousie, déchiré entre son amour pour son père, la crainte mêlée de fascination qu'il éprouve pour sa mère, et sa jalousie elle aussi teintée d'amour envers son chétif demifrère. Loin de la tradition narrative, dont l'auteur n'a retenu, comme s'il s'agissait d'un substrat mythique, que la généalogie perturbée, il n'est question ici ni de conquêtes, ni de Table ronde, ni de géants à terrasser, ni de princesse lointaine appelant au secours. Tout se joue dans cet étroit quadrilatère familial. Elisabeth E. Wein n'écrit pas cependant dans la complète ignorance de la doxa arthurienne, loin s'en faut. Elle ne fait qu'explorer plus avant la thématique de l'inceste fatal, que Malory plaçait déjà au cœur de son roman. Par ailleurs, s'il semble que Gouvaine, la jumelle, soit un personnage d'invention, en revanche Lohot est attesté par la tradition : dans Perlesvaus, il est le fils illégitime de Lysanor et d'Arthur, et il devient, dans Lanzelet, fils de Guenièvre. Elisabeth E. Wein témoigne ici de l'aptitude de ces écrivains pour la jeunesse à puiser dans le fonds arthurien la matière à leurs inventions romanesques, de façon à imbriquer étroitement, à la manière médiévale, héritage et création.

16 Une fois posés les éléments nécessaires à la reconstitution du schéma familial, le prologue laisse la place à l'autobiographie de Mordred, dont on comprend, tardivement, qu'elle s'adresse à sa propre mère, sœur de son père, qu'il appelle " marraine ", et à laquelle il voue des sentiments contradictoires. Le récit tout entier retrace l'itinéraire du jeune garçon de la haine à l'acceptation de soi, dans le rejet absolu de l'inceste et de toute l'ombre qu'il jette sur les êtres.

$17 \mathrm{Au}$ moment où commence ce récit à la première personne, Mordred est un jeune homme fait. De retour à Camelot, il se voit confier par Arthur la responsabilité des mines de cuivre, et est admis dans les secrets du gouvernement. Les jumeaux, quant à 
eux, ont quatorze ans. Lohot est maladif ; sans l'intervention de Mordred, il mourrait à plusieurs reprises. C'est un adolescent remarquablement doué à l'épée ; il est beau, et le peuple l'adore. Mais le retour de Mordred au foyer du père attise les haines. Lohot jalouse Mordred d'être à ce point apprécié d'Arthur, pour sa sagesse et ses compétences. En retour Mordre le bâtard jalouse Lohot le fils légitime. Par ailleurs, au fait du secret honteux de sa naissance, il est dévoré de culpabilité. L'intervention de Morgause suffit à ruiner l'équilibre instable maintenu par le sage Arthur. Attisant les rancœurs de Mordred, Morgause en effet le persuade de capturer et de tuer Lohot ; elle cherche ainsi à se venger de son frère, qui l'a bannie du royaume. Le dernier tiers du roman est consacré à la cruelle expédition hivernale que, sous couvert de chasse, Mordred organise dans l'idée de sacrifier Lohot. Ce sera l'occasion d'un poignant faceà-face entre les deux frères, Mordred renonçant in extremis à tuer, parce que les paroles d'amour de l'adolescent auront désamorcé et sa haine et sa rage de victime innocente :

Et pourtant il avait triomphé de moi de la plus inattendue des manières, en me donnant ce que je n'aurais jamais osé espérer. C'était bien sa confiance, son amour inconditionnel que je convoitais sans le savoir, infiniment plus forts et plus réparateurs que la peur que j'avais voulu lui inspirer. (p. 278)

Le Bâtard du roi Arthur est ainsi un roman sur l'inceste et le secret, sur la jalousie, sur la légitimité. Si le récit s'éloigne de temps à autre de la tradition arthurienne, ce n'est jamais pour longtemps : quelques jalons viennent régulièrement rappeler le contexte arthurien, qu'il s'agisse de Morgause amenant à la cour ses autres fils, Gauvain, Engravain, Gueréhés et Gaheriès, ou encore de l'évocation de la mère d'Arthur, Igraine, qui s'est retirée dans le sud du pays. En revanche, dès que l'ancrage arthurien s'efface, l'auteur s'attache à mettre en avant la dimension antique du contexte diégétique, comme si l'authenticité de l'un compensait les libertés prises par rapport à l'autre. Le royaume arthurien tel qu'il nous est présenté ici est en effet imprégné d'une atmosphère crépusculaire de fin d'empire. Ce n'est pas un vaste royaume, nouvellement conquis et peuplé de chevaliers conquérants à l'ardeur juvénile: le domaine d'Arthur est étroit; Camelot est une ruine sans confort; et l'ensemble de ses possessions laisse une impression de décadence, le sentiment d'une perte irrémédiable en termes de techniques et d'art. Mordred passe ainsi une partie de son été à restaurer les bains antiques, ornés de mosaïques désormais incompréhensibles, qui agrémentaient jadis la résidence. Le monde arthurien n'est donc pas un monde neuf: c'est une société repliée sur elle-même, un clan frustre qui garde le souvenir d'une splendeur passée. Elisabeth $\mathrm{E}$. Wein a réussi à réinjecter dans son roman la conscience d'une strate antique antérieure à l'univers arthurien. Ce choix de dédaigner l'habituel ancrage féodal de la société arthurienne aurait pu n'être qu'une fantaisie esthétisante, un goût de la ruine post-romantique. Bien au contraire ici, il sert le propos de l'auteur à un point tel que l'écoulement des siècles lui-même en vient à être repensé en fonction du drame que vivent ses personnages.

19 Mordred, que sa naissance illégitime tient irrémédiablement à l'écart du pouvoir, malgré ses compétences et la confiance de son père, endure un martyre permanent, parce qu'il connaît le secret honteux de sa conception :

Cette nuit unique, tache sordide dans la vie de mon père, n'est rien en comparaison

de cette lumière noire qui m'accompagne, et qui est ma honte. (p. 53)

20 Au-delà d'une simpliste réhabilitation du personnage de Mordred, Elisabeth E. Wein restitue au personnage une dimension tragique, en le montrant victime: le roman insiste en effet beaucoup moins sur la faute que constitue l'inceste, que sur la 
souffrance de celui qui va naître de cette union inadmissible. En présentant ainsi la situation du point de vue de l'enfant, le roman rétablit l'ordre des responsabilités. Et si Mordred, torturé par la crainte d'être rejeté, accède au désir de sa mère et capture Lohot, c'est moins en définitive pour contenter Morgause que pour forcer son père à admettre sa culpabilité - ce faisant, il libérera Mordred de ce fardeau qu'il endosse malgré lui :

Je veux entendre Arthur dire une chose, une seule chose. Je veux qu'il admette devant tout le monde que c'est sa propre iniquité qui m'interdit de régner. Que la honte est sienne, et non mienne. (p. 223)

21 À la jalousie évoquée dès le prologue, se substitue donc, au cours du récit, un autre moteur de l'action pour Mordred: enfant du tabou, il porte la faute de ses géniteurs comme une culpabilité mortifère - au point qu'il se sent capable d'enfermer autrui dans la même culpabilité, comme si cela avait le pouvoir de l'en décharger :

Je la regardai droit dans les yeux, perplexe, incapable de la croire si naïve. Puis je saisis son visage dans mes mains brûlantes de fièvre et je l'attirai vers moi pour que nous soyons face à face. Mes mains descendirent le long de son cou, de ses épaules et jusqu'à sa poitrine. Un geste suffit pour qu'elle comprenne.

«Je suis votre sœur, dit-elle.

- Vous voyez comment les choses arrivent ", répliquai-je en la lâchant. (p. 224)

Mordred apparait ici enfermé dans un passé qui n'est pas le sien. La symbolique de l'Antiquité, dont les traces affleurent à tous les niveaux de ce royaume arthurien décadent, prend alors tout son sens. Mordred en effet est prisonnier du passé : sinon le sien, du moins celui de ses parents. Très cultivé, il est celui qui veut donner du sens aux traces de ce passé. Un jour qu'il explore une des galeries de la mine, il découvre une caverne ornée d'une scène de chasse primitive, qui l'émerveille et l'obsède ; consigné dans sa chambre, il lit la poésie romaine, les quelques pièces grecques rescapées dont il possède une traduction latine; et, aux enfants hébétés qui contemplent les mosaïques de l'atrium sans en identifier les motifs, il explique la symbolique du tétramorphe et raconte l'Apocalypse. L'omniprésence d'un passé antique, archaïque, voire préhistorique, signale ici qu'on n'échappe pas à son passé. Et si les fils de Morgause peuvent se contenter d'observer les motifs des mosaïques romaines sans en comprendre le sens, Mordred, qui est en quête de sa propre identité et de sa place dans l'existence, doit, comme en témoignent ses efforts pour décrypter le passé culturel, s'approprier son propre passé, pour être capable d'assumer ses propres erreurs, et de rejeter les culpabilités qui ne lui reviennent pas.

Le monde arthurien d'Elisabeth E. Wein est antique et décadent, c'est une ruine inconfortable et précaire, tant que les personnages qui le peuplent ne font pas la paix avec leur propre passé. Il faut à Mordred le détour par un simulacre de Grande Chasse archaïque - la même que celle qui figure sur les parois de la galerie souterraine --, dans laquelle Lohot serait la victime expiatoire, pour parvenir à formuler les désirs qui l'empoisonnent : que son père reconnaisse ses responsabilités; que son jeune frère lui offre son amitié. Rien d'étonnant à ce que le personnage ne puisse résoudre son drame intime que par le recours à un motif mythique. Le contexte antiquisant n'a-t-il pas suffisamment souligné le lien entre l'univers arthurien évoqué et la culture antique de tradition gréco-romaine? Et Arthur, qui se lie à Morgause alors qu'il ignore encore qui étaient ses parents, n'est-il pas comme Oedipe la victime d'un destin qui le dépasse ? Ainsi, de la même façon que les mythes, depuis Freud, appellent à lire, dans les destinées familiales de héros antiques, le sens de nos propres démêlés affectifs, de 
même le roman arthurien d'Elisabeth E. Wein, parce qu'il entretient un lien ténu mais jamais rompu avec le monde de la sagesse antique, mais aussi avec un paganisme au résonances nouvelles, invite à remettre en perspective l'aventure arthurienne. Réduite aux dimensions d'une cellule familiale, elle prend pourtant une ampleur considérable, par les notions qu'elle met en jeu. Mordred, longtemps prisonnier d'un passé entaché par la transgression, parvient à s'en libérer par le détour de la mise en scène mythique : celle de la Grande Chasse mythique, qui devait être mise à mort de son frère, et qui est réconciliation; celle de l'Apocalypse où, à l'occasion de la découverte des motifs ornant l'atrium, sa mère le pare comme le Ressuscité des textes bibliques (elle cite l'Apocalypse $1-12$, p. 188) ; celle de la renaissance cyclique des saisons, jouée par les paysans déguisés lors du Solstice d'hiver. Ainsi délivré, il peut accepter le pardon de son père, et s'engager enfin dans la voie de la maturité, loin des illusoires régressions souterraines, loin aussi de la séduction malsaine de sa mère :

Mon père, homme de justice et de pardon. Il s'avança, me prit les mains et m'aida à me relever.

« Je t'ai dit un jour, t'en souvient-il ?, que tu pourrais toujours revenir vers moi. »

Et voilà, Marraine, pourquoi je ne pourrai plus jamais revenir vers vous. (p. 286)

La chute du roman consacre donc le retour à un équilibre d'amour et de pouvoir au sein de l'étroite cellule familiale. Renonçant au brouillage des strates générationnelles induit par l'inceste, Mordred assume sa place dans la filiation, en faisant allégeance à Lohot. Il retrouve ainsi sa place, dans la famille comme dans le royaume.

Curieusement, l'auteur semble brusquement dénier au roman toute postérité. D'ailleurs, Arthur ne meurt pas ici de la main de Mordred; il n'y a donc pas de renaissance arthurienne possible, qui introduirait la pérennité au sein d'une histoire pourtant aboutie. Elisabeth E. Wein, en bouleversant le dénouement de son récit, procède ici à une abolition de la notion même de cycle, au profit d'un resserrement sur la situation concrète des personnages, qui vivent eux-mêmes au sein d'un autre cycle, biologique et rituel. Le roman se clôt d'ailleurs sur cette phrase, empruntée à la mise en scène païenne du solstice d'hiver :

La nouvelle année pouvait commencer. (explicit)

Ce roman est donc un tour de force. Partant d'une situation empruntée au cycle (Mordred fils incestueux d'Arthur), puis brodant autour, comme la tradition elle-même l'y autorise, il renverse totalement les rôles: Mordred ne tue pas Arthur, il lui fait allégeance et Arthur lui pardonne ses fautes. Le Bâtard du roi Arthur est donc un cas limite de ce qu'on peut nommer " enfance » arthurienne, tant sont grandes les libertés finalement prises avec la tradition romanesque. De cette entorse aux lois du genre naît paradoxalement le plus fort des récits pour enfants envisagés pour cette étude. Parce qu'au-delà de l'aménagement romanesque d'interstices temporels, il mène une réflexion sur la notion même de personnage. Mordred n'a jamais existé, pas davantage qu'Arthur ou Guenièvre: ce que nous savons de lui n'est que littérature, parole figée par l'écrit, qui le constitue en être de papier. Or que ces paroles se mêlent à d'autres, que Malory croise l'Apocalypse de Jean ou la poésie antique, et voilà le personnage aiguillé sur de nouvelles voies, aussi légitimes littérairement que l'étaient celles qu'inventaient en leur temps Chrétien de Troyes et ses continuateurs. Le roman est une réflexion sur ce qui constitue la liberté fondamentale de l'être, à savoir sa capacité à inventer son existence, dans l'acceptation de ses origines mais sans inféodation à ce que celles-ci pourraient sembler prescrire. Mais il est aussi, en actes, une manifestation de cette même liberté, cette fois-ci créatrice, qui dans l'ordre du littéraire réside dans 
l'invention et la reprise, sans cesse mêlées, du « jamais écrit » et du « déjà lu ». Elisabeth E. Wein affirme, après Borgès, qu'on n'écrit jamais que parce que tant d'autres livres ont été écrits avant le nôtre. Seulement, elle le dit aux enfants. Et cela ne s'était jamais fait.

\section{NOTES}

1.Elzbieta, Histoires d'enfances, Éditions du Rouergue, 2003, p. 7.

2.Un colloque sur les « enfances arthuriennes » s'est justement tenu à Rennes en mars 2003, dont les actes sont à paraître.

3. « Le récit d'enfance et ses modèles : colloque de Cerisy-la-Salle (27 septembre- $1^{\mathrm{e}}$ octobre 2001) », Collectif.

4.Claude Sylvain (pseudonyme de Jeannine Harmand), Contes et légendes du roi Artus, L'Enfance de Merlin, ill. F. Nathanson, Paris, Gautier-Languereau, 1947.

5.Le volume remanié contient les quatre textes alors terminés: The Sword in the Stone (1939), The Witch in the Wood (1940), The Ill-made Knight (1941), The Candle in the Wind (1958). Un volume posthume sera publié en 1977 : The Book of Merlyn.

6.T. H.White, L'Epée dans le roc, trad. Jean Muray, ill. Georges Beuville, Paris, Hachette, Bibliothèque verte, 1965.

7.Art est surnommé « the Wart », par proximité phonique, ce qui signifie « la verrue »; la traduction de Jean Murray préfère le surnom « Moustique »; celle, plus récente, de Monique Le Bailly restitue la dimension sémantique mais oublie le jeu de rime en appelant son personnage « La Verrue ».

\section{AUTEUR}

\section{CÉCILE BOULAIRE}

Université de Tours 